Article

\title{
Análise da Variabilidade Espaço-Temporal do SPI: Um Estudo de Caso para a Sub-Bacia Choró, Ceará, Brasil
}

\author{
Greicy Kelly da Silva ${ }^{1}$ (D), Antônio Duarte Marcos Júnior ${ }^{1}$, Carlos Eduardo Sousa Lima ${ }^{1}$, \\ Marx Vinicius Maciel da Silva ${ }^{1}$, Cleiton da Silva Silveira ${ }^{1}$, \\ Emerson Mariano da Silva ${ }^{2}$ (D), Ilma Ribeiro de Lima ${ }^{2}$ \\ ${ }^{1}$ Programa de Pós-Graduação em Engenharia Civil, Departamento de Engenharia Hidráulica e \\ Ambiental, Universidade Federal do Ceará, Fortaleza, CE, Brasil. \\ ${ }^{2}$ Mestrado Profissional em Climatologia e Aplicações nos Países da CPLP e África, Universidade \\ Estadual do Ceará, Fortaleza, CE, Brasil.
}

Recebido em: 31 de Janeiro de 2021 - Aceito em: 16 de Abril de 2021

\begin{abstract}
Resumo
Este trabalho teve como objetivo avaliar a variabilidade espaço-temporal do Índice Padronizado de Precipitação (SPI), de forma a identificar períodos de escassez/excesso hídrica (o) na Sub-Bacia Choró no Estado do Ceará. Para tanto, trabalhou-se com uma série histórica de dados pluviométricos para o período de 1974 a 2018 e com o SPI nas escalas de 3, 6, 12 e 24 meses. Uma acentuada variabilidade interanual foi observada, apontando de 2012-2018 a maior ocorrência de eventos secos, sendo estes melhor representados, nas escalas interanuais, em intensidade e duração quando comparado com as escalas (intras)sazonais. Também constatou-se que os períodos contendo os eventos secos (chuvosos) de maior intensidade, apresentados nas escalas 3, 6 e 12 meses do SPI, coincidiram, na maioria dos casos, com períodos de $E l$ Niño Oscilação Sul e La Niña. Em suma, o SPI se mostrou uma ferramenta poderosa ao quantificar os eventos secos e chuvosos na Sub-Bacia Choró, podendo ser útil no monitoramento do déficit hídrico em escalas temporais de curto, médio e longo prazo.
\end{abstract}

Palavras-chave: SPI, Choró, El Niño, escassez hídrica.

\section{Analysis of SPI Spatiotemporal Variability: A Case Study for the Choró Sub- Basin, Ceará, Brazil}

\begin{abstract}
This work has as objective to evaluate the spatiotemporal variability of the Standardized Index of Precipitation (SPI), in order to identify periods of water scarcity/excess in the Choró Sub-Basin in the State of Ceará. For this purpose, we worked with the historical series of rainfall data for the period from 1974 to 2018 and with the SPI on scales of 3, 6, 12, and 24 months. A pronounced inter-annual variability was observed, pointing out from 2012-2018 the highest occurrence of dry events, being these better represented, in the inter-annual scales, in intensity and duration when compared to the intras-seasonal scales. It was also found that the periods containing the most intense dry (rainy) events, presented on the scales 3, 6, and 12 months of SPI, coincided in most cases with periods of El Niño Oscilação Sul and La Niña. In short, SPI proved to be a powerful tool when quantifying dry and rainy events in the Choró Sub-Basin and can be useful in monitoring the water deficit, in short, medium, and long term time scales.
\end{abstract}

Keywords: SPI, Choró, El Niño, water scarcity.

\section{Introdução}

Nos últimos anos eventos extremos, como secas severas, aumentaram em diversas regiões do mundo (Allen et al., 2012). Essas secas impactam negativamente diversos setores: agricultura, pecuária, sistemas hídricos de armazenamento e abastecimento, indústria, energia, dentre diversos outros. O Nordeste do Brasil (NEB), e em especial o Estado do Ceará (CE), são regiões conhecidas

Autor de correspondência: Greicy Kelly da Silva, greicy.silva@alu.ufc.br. 
por sofrerem com longos períodos de estiagens, como a que a ocorreu no período de 2012 a 2018 (Marengo et al., 2020; Pontes Filho et al., 2020). Além disso, a região, que está localizada em grande parte na região semiárida, caracteriza-se por uma grande variabilidade espacial e temporal das chuvas (Marengo et al., 2020). Nesse contexto, surge a necessidade de conhecer os padrões de secas extremas quanto a sua variabilidade temporal e espacial sob o clima presente, de forma a auxiliar os órgãos gestores na tomada de decisão quanto a orientação de avaliações e medidas de vulnerabilidade e adaptação.

Identificam-se as secas após um longo período com déficit de precipitação ou sem nenhuma precipitação (Vicente-Serrano et al., 2010). Diversas definições e ferramentas matemáticas para quantificar, monitorar e analisar as secas foram desenvolvidas no último século. Uma importante ferramenta desenvolvida em 2014 para a região Nordeste foi o monitor de secas, coordenado pela Agência Nacional de Águas (ANA) com o apoio da Fundação Cearense de Meteorologia e Recursos Hídricos (FUNCEME) e produzido por um grupo formado por diversas instituições e pessoas de forma totalmente colaborativa e voluntária. Atualmente, o monitor permite comparar mensalmente a evolução das secas em 20 unidades da federação, de forma integral nas regiões Nordeste, Sul e Sudeste, a partir de dados de chuva, de níveis de reservatórios, de umidade de solo, entre outras informações. Estas são então combinadas e discutidas, seguindo-se então para o desenho e validação do mapa de monitor de secas da região em análise (ANA, 2019).

Outra ferramenta bastante usada é o Índice de Precipitação Padronizado (SPI)-do ínglês Standarized Precipitation Index, formulado no Colorado Climate Center por Mckee et al. (1993). Tem como vantagem depender apenas da precipitação como variável de entrada. Além disso, o SPI quantifica as secas em diversas escalas temporais podendo ser usado nas estações seca e chuvosa (Hayes et al., 1999).

No Brasil, o SPI vem sendo utilizado em diversos trabalhos para diferentes regiões do país, mostrando-se uma ferramenta poderosa na detecção de eventos extremos chuvosos/secos (Gois et al., 2015; Uliana et al., 2015; Sousa et al., 2016; Santos et al., 2017; Bonfim e Da Silva, 2018). Ademais, alguns estudos recentes utilizando o SPI como os de Carmo e Lima (2020), Da Silva et al. (2020), Silva et al. (2019), Nascimento et al. (2017) e Nascimento et al. (2015), vêm sendo realizados também especialmente sobre a região do NEB.

Nesse contexto, o objetivo deste trabalho é analisar a variabilidade espaço-temporal dos principais eventos secos e chuvosos na Sub-Bacia Choró, no período de 1974 a 2018, bem como identificar a presença de escalas temporais destes eventos relacionadas com eventos El Niño Oscilação Sul (ENOS) e Dipolo do Atlântico.

\section{Materiais e Métodos}

\subsection{Região de estudo}

A área de estudo compreende a Sub-Bacia Choró que ocupa uma área de $4.717,11 \mathrm{~km}^{2}$ e está contida a região hidrográfica que engloba as Bacias Metropolitanas, sístema hídrico de importante destaque no Estado do Ceará, pois atende às demandas do abastecimento humano e indústrias da Região Metropolitana de Fortaleza. A SubBacia Choró, possui o rio Choró, de aspecto bastante intermitente, como elemento de maior destaque em sua drenagem (CEARÁ, 2016). Quanto a classificação climática de Köppen-Geiger (1936), caracteriza-se com clima tropical com estação seca de verão. Prevalece em grande parte da região da bacia o clima semiárido, com seis meses secos e apresentando temperatura quente com a média superior a $18{ }^{\circ} \mathrm{C}$ ao longo de todo o ano (IBGE, 2002). A Fig. 1 apresenta o mapa de localização da região supracitada.

\subsection{Dados utilizados}

Os dados interpolados de precipitação considerados no presente estudo são provenientes da FUNCEME tendo sido previamente selecionados os postos pluviométricos contidos dentro da área de estudo. Os dados são interpolados para uma série histórica de 1974 a 2018 , em grade regular de $0.5 \mathrm{~km}$ utilizando a técnica de krigagem.

\subsection{Cálculo do SPI}

O SPI pode ser calculado em diferentes escalas de tempo. Na escala de 1 mês (SPI-1) reflete condições de curto prazo e pode ser aplicado a alterações na umidade do solo e na agricultura. O SPI de 3 meses (SPI-3), por sua vez, reflete condições de médio e curto prazo tendo grande aplicação na análise de umidade do solo. A médio prazo, toma-se as escalas de 6 meses (SPI-6) e 9 meses (SPI-9) que apontam tendências de médio prazo nos padrões de precipitação, este último é um bom indicador de impactos siginificativos de seca na agricultura. E o SPI de 12 meses ou escalas superiores reflete os padrões de precipitação a longo prazo, geralmente vinculados às vazões médias, níveis de água de reservatórios, e níveis de água subterrânea (Albuquerque, 2010; Zargar et al., 2011). Os trabalhos de Guttman (1999) e Fernandes et al. (2009) trazem mais detalhes sobre o algoritmo de cálculo do índice SPI. Neste trabalho, o SPI foi calculado, em programa R, escolhendo-se as escalas temporais SPI de 3 meses (SPI-3), 6 meses (SPI-6), 12 meses (SPI-12) e 24 meses (SPI-24) (R Core Team, 2020). Os valores de SPI podem ser interpretados para categorizar a seca ou umidade, de acordo com McKee et al. (1993, 1995) conforme os intervalos: $\leq-2.0$ : Seca Extrema; -1.5 a -1.99: Seca Severa; -1.0 a -1.49 : Seca Moderada; -0.99 a +0.99: Normal; 1.0 a 1.49: Umidade 


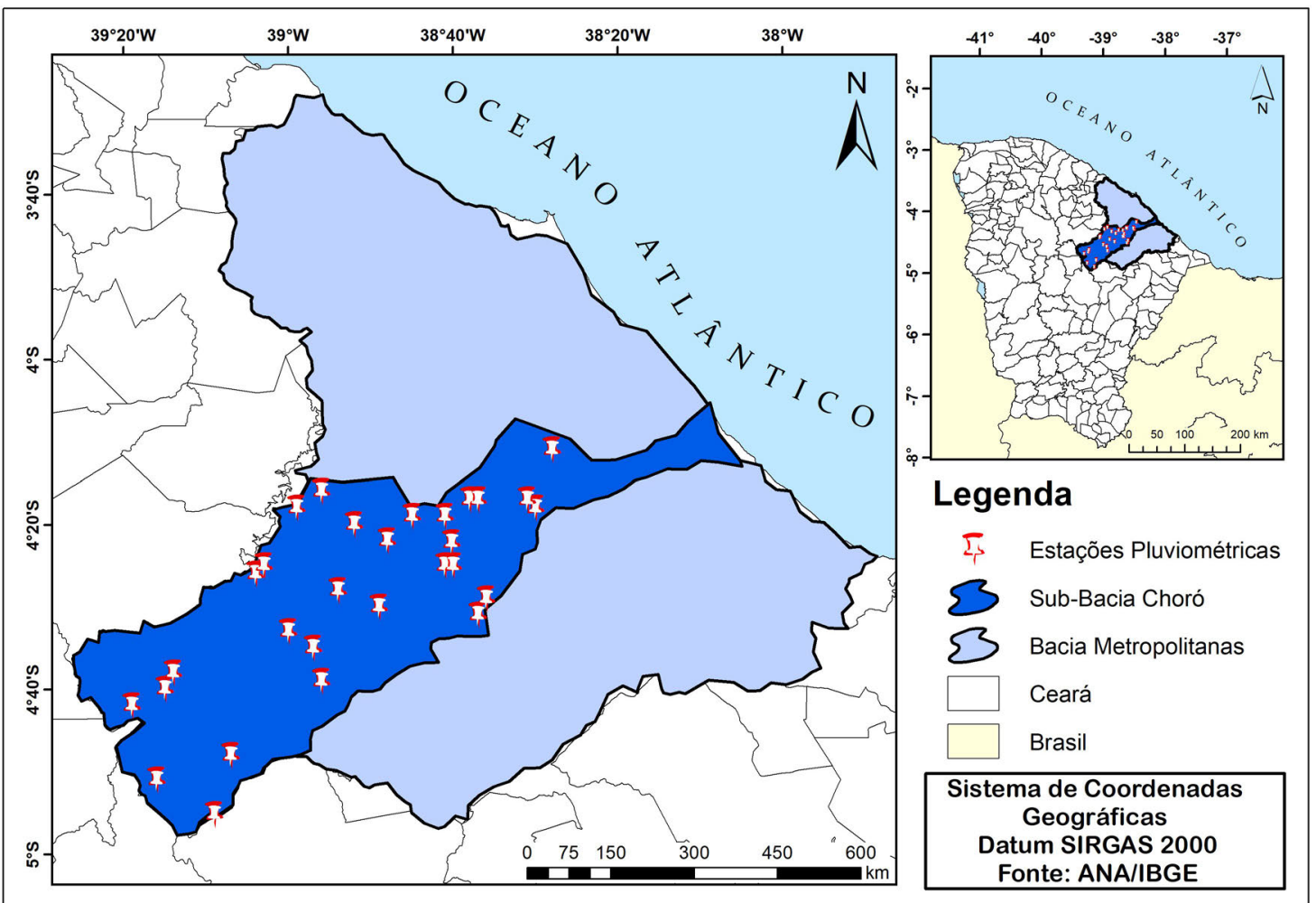

Figura 1 - Localização da Sub-Bacia Choró.

Moderada; 1.5 a 1.99: Umidade Severa e $\geq 2.0$ : Umidade Extrema. Seguindo outros estudos, neste trabalho uma sequência de valores negativos de SPI indicará uma condição de seca bem como uma sequência de valores positivos, indicará um período de umidade (Aghakouchak et al., 2015; Brito et al., 2018).

\subsection{Zona de Convergência Intertropical e o Dipolo do Atlântico Tropical}

A Zona de Convergência Intertropical (ZCIT) é o principal sistema atmosférico influenciador das chuvas atuante sobre o norte do NEB/CE (Reboita et al., 2014). Sua localização, no entanto, sofre a influência dos gradientes meridionais de anomalias da Temperatura da Superfície do Mar (TSM) propiciados por um padrão de anomalias de TSM existente no Oceano Atlântico. A esse padrão denomina-se Dipolo do Atlântico Tropical caracterizado por suas duas vertentes: Dipolo Positivo (DP), quando ocorrem anomalias positivas no Atlântico Norte e anomalias negativas no Atlântico Sul; Dipolo Negativo (DN), quando ocorrem anomalias positivas no Atlântico Sul e negativas no Atlântico Norte (Nobre e Shukla, 1996). As áreas doravantes denominadas TNAI (Atlântico Tropical Norte) e TSAI (Atlântico Tropical Sul) se referem à região associada ao padrão de Dipolo do Atlântico Tropical, nas áreas compreendidas entre $0-20^{\circ} \mathrm{S}$ e $10^{\circ}$
E- $30^{\circ} \mathrm{W}$ e entre $5^{\circ} \mathrm{N}-23,5^{\circ} \mathrm{N}$ e $15^{\circ} \mathrm{W}-57,5^{\circ} \mathrm{W}$, respectivamente.

\subsection{El Niño Oscilação Sul e La Niña}

Vários estudos citam a influência de fenômenos atuantes na região do oceano Pacífico Equatorial como possíveis responsáveis pela ocorrência ou não ocorrência de precipitação, entre eles: o ENOS (Tedeschi et al., 2016). O ENOS pode ser definido como uma grande oscilação acoplada de interação oceano-atmosfera de grande escala, caracterizada por anomalias positivas da Temperatura da Superfície do Mar (TSM) e Pressão ao Nível Médio do Mar (PNMM) sobre o Oceano Pacífico, além do enfraquecimento dos ventos alísios de oeste. Um padrão oposto é observado em períodos de La Niña, com anomalias negativas de TSM no Oceano Pacífico e intensificação dos ventos alísios de oeste. Os episódios desses fenômenos duram em média de 9 a 12 meses (NOAA, 2017). Segundo Trenberth e Hoar (1997) a região chave para interações acoplado oceano-atmosfera para o ENOS se encontra mais a oeste $(5 \mathrm{~N}-5 \mathrm{~S}, 170 \mathrm{~W}-120 \mathrm{~W})$-região do índice Niño 3.4-que vem sendo utilizado por trabalhos mais recentes através do Oscilation Niño Index (ONI)médias trimestrais das anomalias de TSM sobre essa região (Alizadeh-Choobari, 2017; Singh et al., 2017). A Fig. 2 traz os valores do ONI para o período de 20152018. 


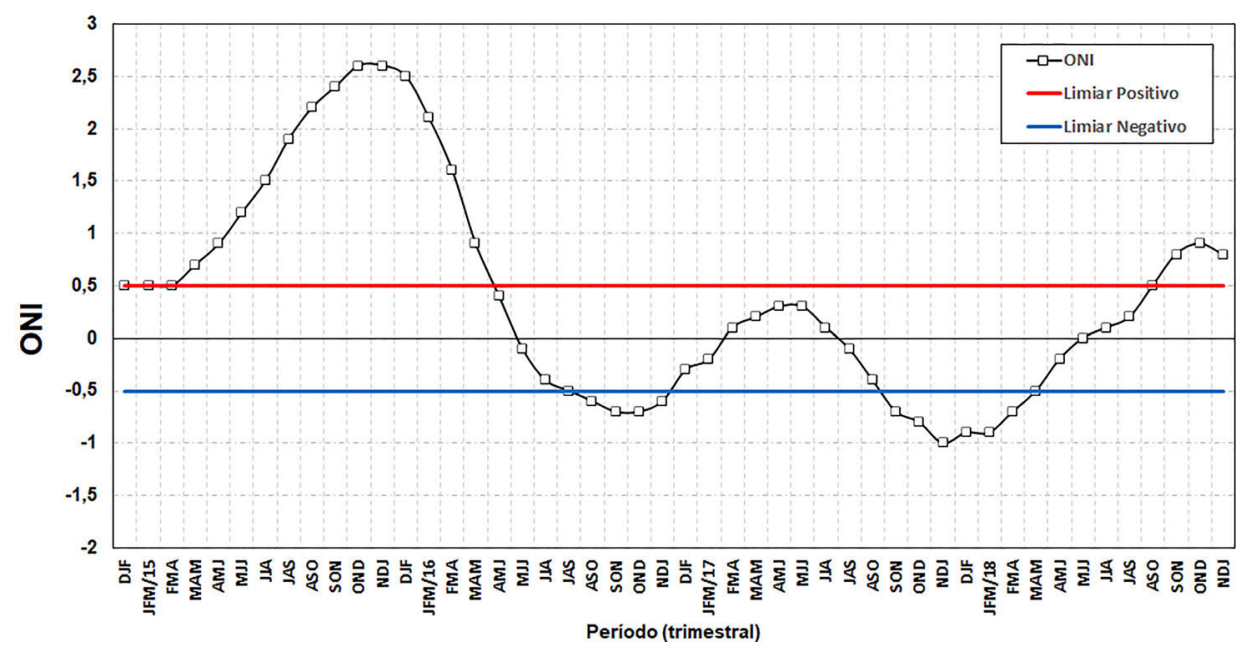

Figura 2 - Anomalias da TSM referente ao ONI de 2015-2018. As linhas tracejadas em vermelho e azul são os limiares positivos e negativos, respectivamente. Fonte: Adaptado do NOAA (2017).

\section{Resultados e Discussões}

\subsection{Identificação dos eventos de secas pelo índice SPI}

As Figs. 3A, 3B, 3C e 3D apresentam o índice SPI de 3 meses (SPI-3), 6 meses (SPI-6), 12 meses (SPI-12) e 24 meses (SPI-24), respectivamente, para a Sub-Bacia Choró no período de 1974 a 2018.
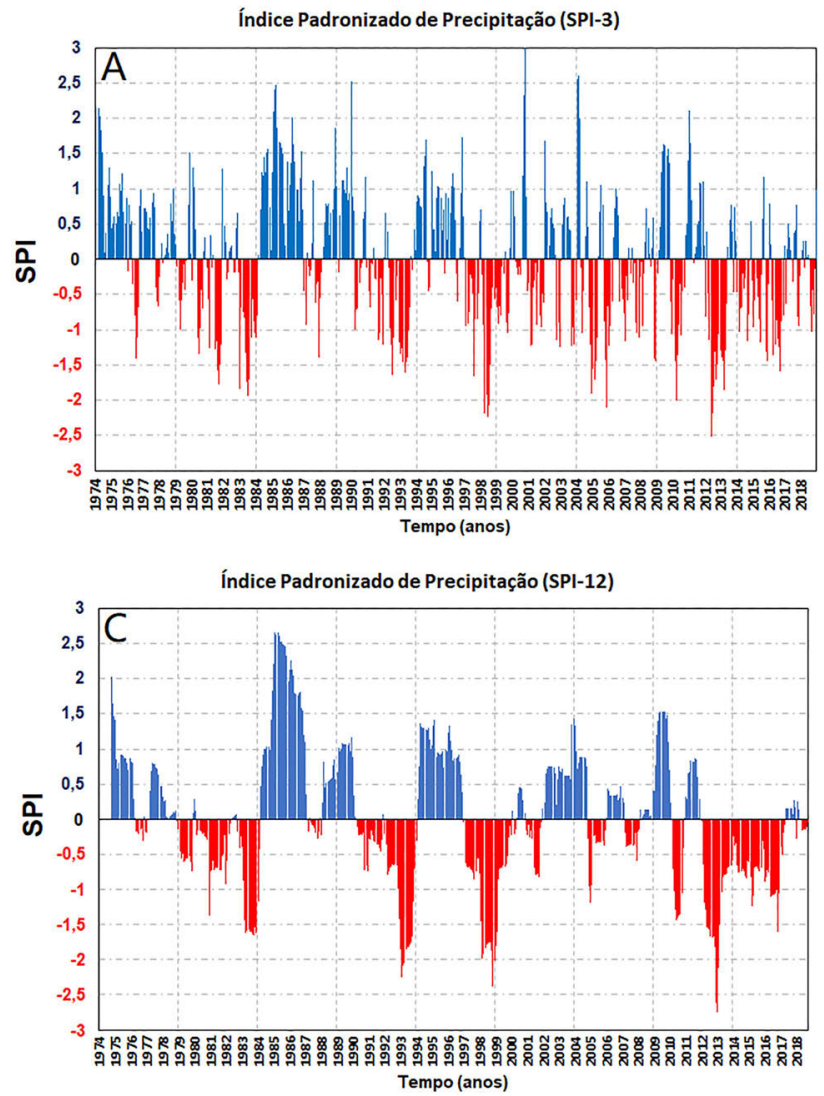

Observa-se a suavização dos períodos identificados com anomalias extremas à medida que aumenta-se a escala temporal, o que pode ser explicado, segundo Mckee et al. (1993), pela própria metodologia que associa-se a média móvel. Nas escalas SPI-3 e SPI-6, os principais eventos de seca aconteceram nos anos de 1983, 1992/ 1993, 1997-1999, 2012/2013, 2015/2016, categorizando
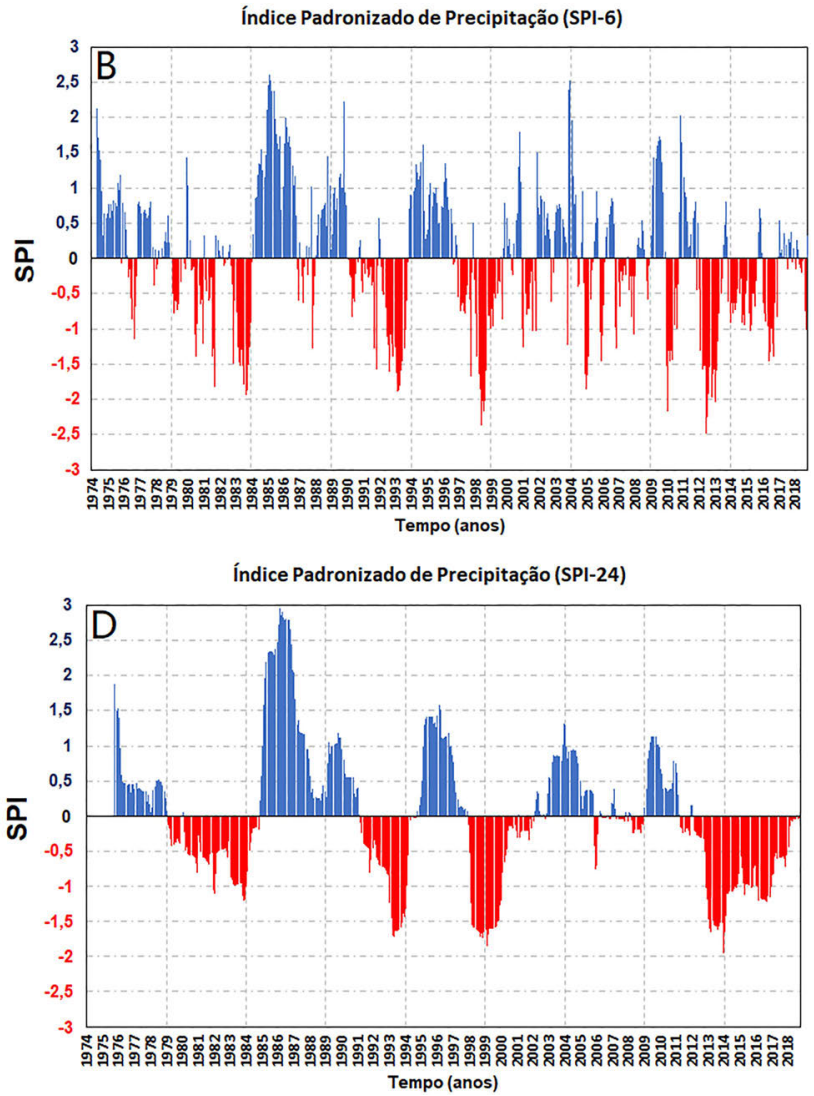

Figura 3 - Índice SPI nas escalas SPI-3 (A) SPI-6 (B) SPI-12 (C) SPI-24 (D), para a Sub-Bacia Choró no período de 1974 a 2018. 
condições Severamente e Extremamente Secas. Nas escalas SPI-12 e SPI-24, se observou uma melhor representação dos episódios de maior intensidade e duração, quando comparados à outros períodos que revelaram menor intensidade e em outras escalas. Na escala SPI-12, identifica-se uma acentuada variabilidade interanual e decadal, sendo o período de 2012 a 2018 o que contém maior ocorrência de eventos intensos de seca. Esse é o período seco mais longo já registrado, conforme reportado na literatura (Marengo et al., 2020; Pontes Filho et al., 2020). Neste período, os valores de SPI-12 registraram um evento de até -2.7 (março/2013), sendo, portanto, o episódio de seca mais extremo identificado na série temporal.

\subsection{Análise da variabilidade interanual e sazonal}

Nesta etapa, optou-se por utilizar os SPI-3, SPI-6 e SPI-12, de forma a avaliar espacialmente a variabilidade interanual e sazonal dos episódios de eventos intensos identificados dentre o período de 2012-2018. Segundo Brasil (2020), os anos 2015/2016 e 2017/2018 registraram, respectivamente, eventos do tipo El Niño forte e La Niña moderada. Dessa forma, foram escolhidos os anos de 2015 a 2018 para se avaliar a variabilidade climática na região da bacia. A Fig. 4 traz a climatologia para o período de 1974 a 2018 sobre a Sub-Bacia Choró. Considerando um recorte temporal de três meses, pode-se observar que os valores máximos de chuva ocorrem durante os meses de fevereiro-abril (período chuvoso) e os mínimos nos meses de agosto-outubro (período seco). A análise espacial do SPI foi realizada para o mês de março que apresenta a maior normal climatológica do período de chuvas.

As Figs. 5, 6 e 7 mostram a variabilidade espacial do SPI, sobre a Sub-Bacia Choró, referente ao mês de março nas escalas de 3, 6 e 12 meses respectivamente.

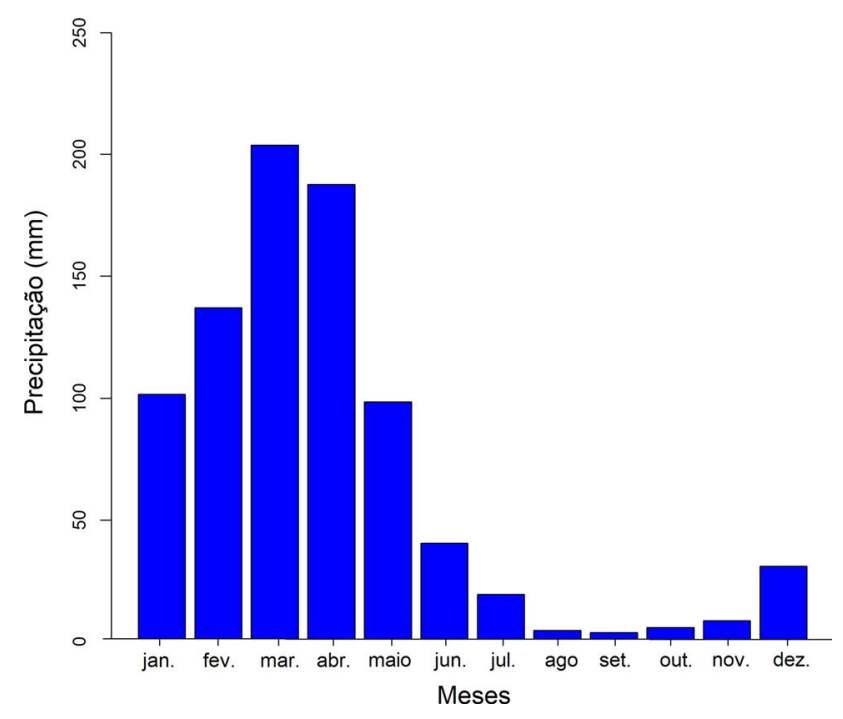

Figura 4 - Série climatológica de precipitação para o período de 1974 a 2018 para a Sub-Bacia Choró.
Observa-se que os episódios de seca evidenciados no SPI-3 e SPI-6 apresentaram menor sensibilidade quanto a intensidade de secas moderadas a extremas quando comparadas a escala anual (Fig. 7). O mesmo foi observado por Nascimento et al. (2017), o que pode ser explicado pelo menor período de acumulação da precipitação considerados no cálculo do SPI na escala intrasazonal.

Considerando a escala interanual, as secas severa e moderada observadas em 2015 e 2016, respectivamente, concordam com os valores positivos do ONI mostrados na Fig. 2. O que reforça, em parte, a influência dos eventos de circulação de grande escala sobre o Ceará. Uma discordância, no entanto, é identificada em março/2017 (ano que se destacou a ocorrência de uma La Niña moderada). Neste, o SPI-12 foi categorizado entre Severamente e Extremamente Seco. De outra forma, a escala intrasazonal de 3 meses mostrou melhor sensibilidade quanto a $L a$ Niña ao categorizar o SPI como Neutro em 2017, e de Umidade Moderada (até mesmo Severa) em 2018 em grande parte da bacia, resultados que coincidem com o período Normal identificado a partir da Fig. 2.

3.3. Condições meteorológicas no Ceará para os anos 2015, 2016, 2017 e 2018

A Fig. 8 apresenta as anomalias de precipitação para o mês de março para os anos a) 2015, b) 2016, c) 2017 e d) 2018. Nota-se que os anos 2016 e 2018 apresentaram os valores mais críticos de anomalias negativas de precipitação, abrangendo toda a Sub-bacia do Choró.

O déficit pluviométrico constatado em março de 2016 justifica-se, sobretudo, pela ocorrência de um evento de El Niño de categoria Forte, o ONI apresentou valores acima de +1.5 nos trimestres janeiro-fevereiro-março (JFM) e fevereiro-março-abril (FMA)-ver Fig. 2, enquanto a TSM apresentou um padrão anômalo de aquecimento nas regiões TNAI e TSAI (ver Fig. 9). Logo, o comportamento anômalo de aquecimento da TSM no TNAI e TSAI identificados na Fig. 9B, pode ter contribuído para uma posição da ZCIT mais ao norte da sua posição climatológica no ano 2016.

Conjuntamente, um pulso desfavorável da Oscilação Intrassazonal Madden-Julian (OMJ) favoreceu a atuação de VCANs, o que certamente influenciou na escassez de chuvas sobre o NEB, conforme boletim publicado pelo Brasil (2016). De outro modo, em março/2018 (período categorizado com uma La Niña Moderada-Ver Fig. 2) a fraca atividade convectiva pôde ser explicada também pelo posicionamento mais ao norte da ZCIT conforme padrão de dipolo promovido no campo de anomalia de TSM, caracterizado por anomalias negativas no TSAI e positivas no TNAI (ver Fig. 9D), aliado a passagem de um pulso subsidente do fênomeno de OMJ sobre o continente sulamericano, segundo o boletim climático do Brasil (2018). Para março/2017, com ONIs dentro da faixa do limiar negativo caracterizando a La Niña de intensidade 

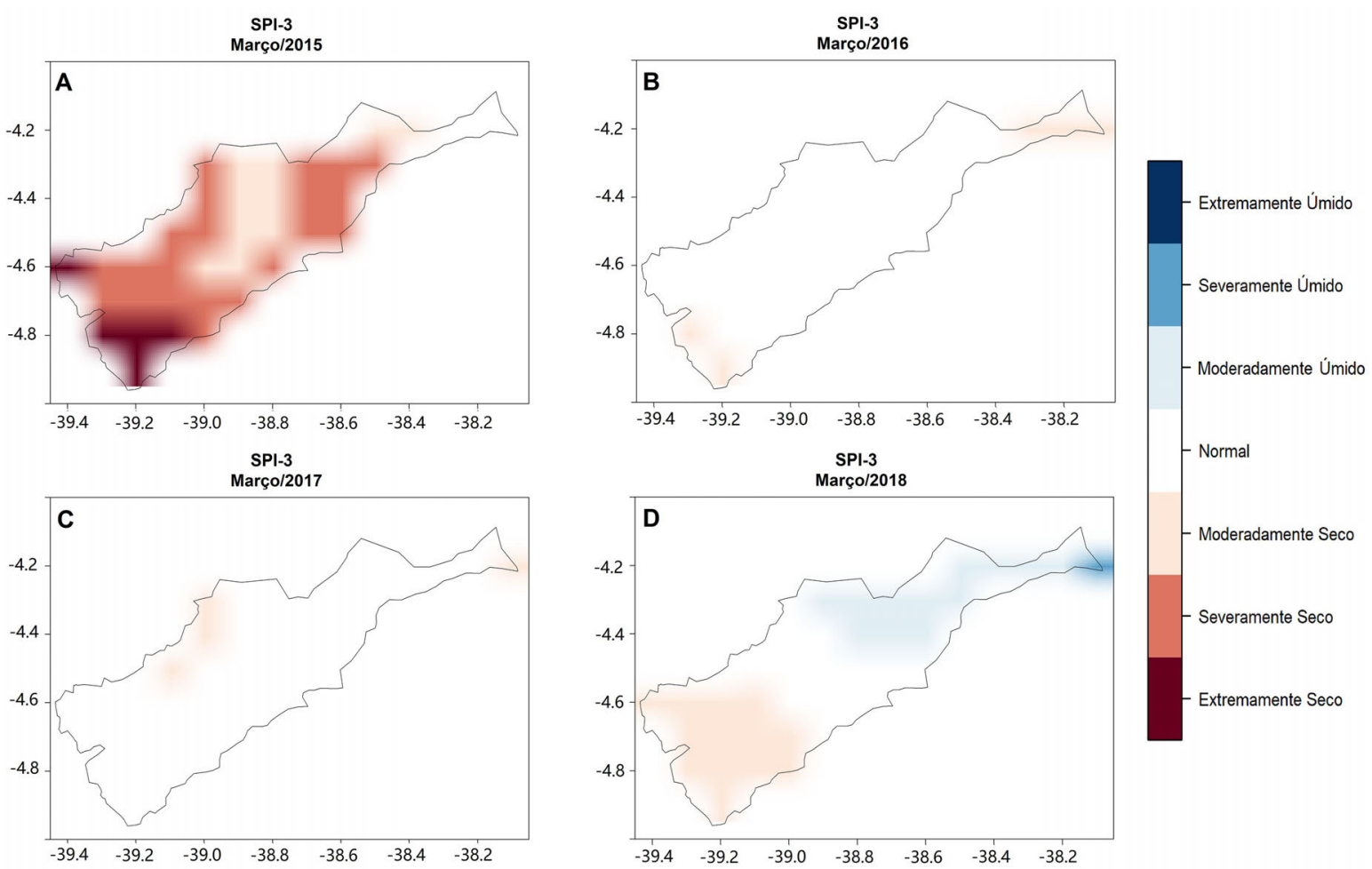

Figura 5 - Variabilidade Espacial do SPI-3 sobre a Sub-Bacia Choró nos anos 2015 (A) 2016 (B) 2017 (C) 2018 (D).
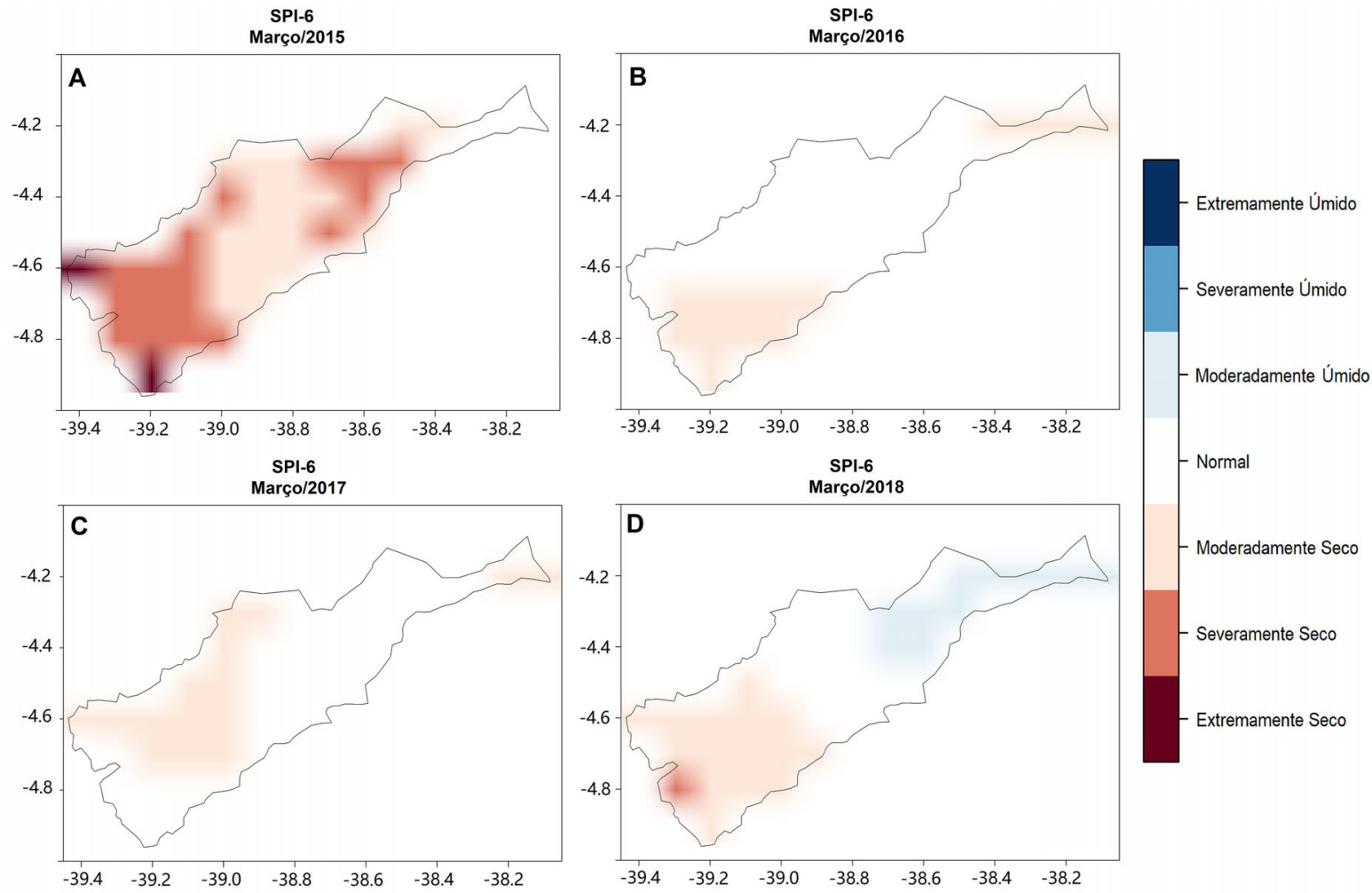

Figura 6 - Variabilidade Espacial do SPI-6 sobre a Sub-Bacia Choró nos anos 2015 (A) 2016 (B) 2017 (C) 2018 (D). 

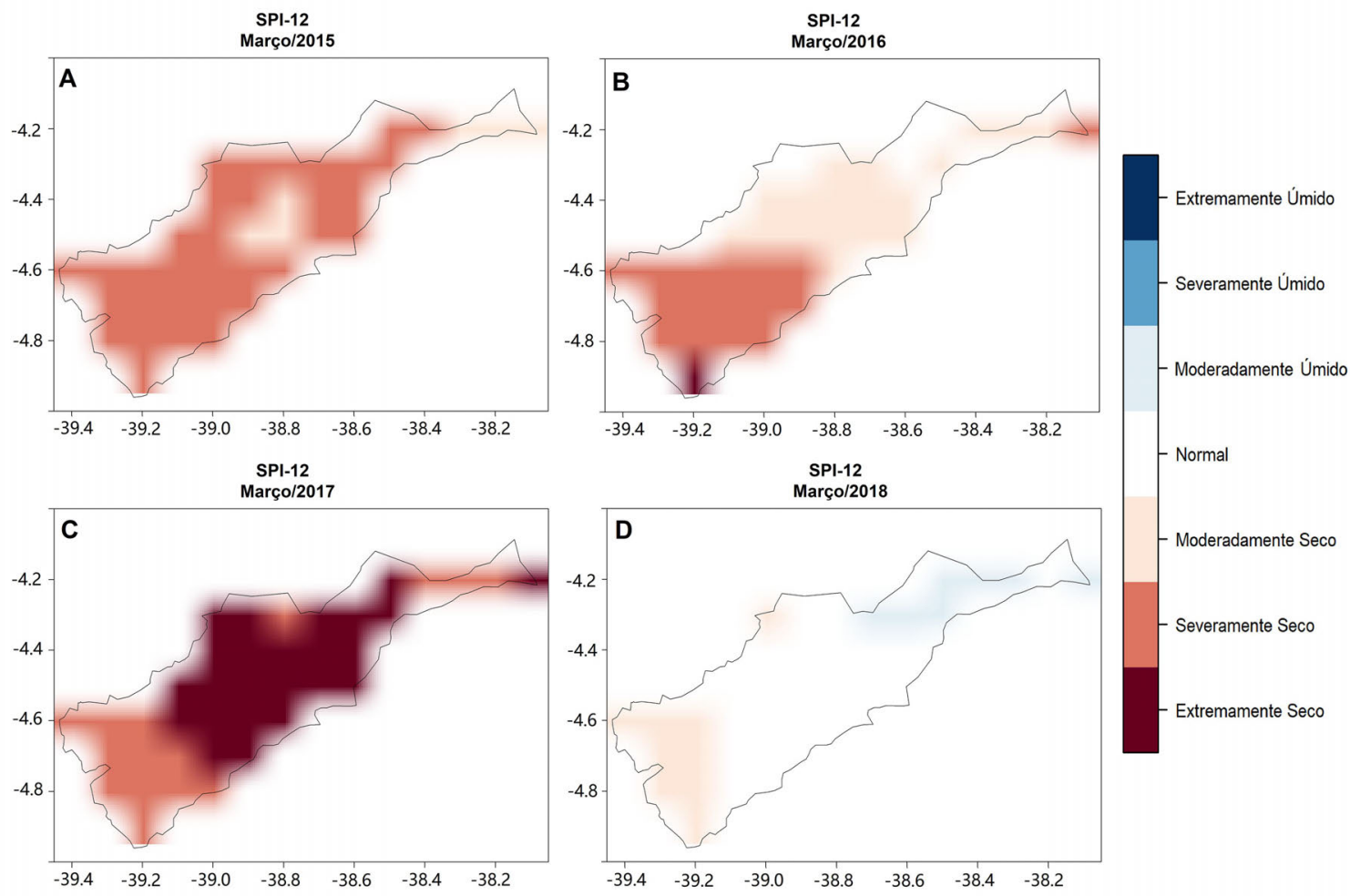

Figura 7 - Variabilidade Espacial do SPI-12 sobre a Sub-Bacia Choró nos anos 2015 (A) 2016 (B) 2017 (C) 2018 (D).

Fraca, pode ter tido sua predominância de chuvas acima da média histórica devido a formação de Linhas de Instabilidade próxima a costa norte da América do Sul aliada à aproximação da ZCIT, conforme mencionado pelo Brasil (2017) em seu boletim do clima. Por fim, para março/ 2015, período que mais revelou uma maior variabilidade espacial dentro da bacia Sub-Choró, sofreu a predominância do fenômeno El Niño Oscilação Sul. As anomalias de TSM , negativas no Atlântico Norte e positivas no Atlântico Sul-ver Fig. 9A, favoreciam o posicionamento da ZCIT mais ao sul da sua posição climatológica. Entretanto, teve sua atuação enfraquecida devido ao enfraquecimento também dos ventos alísios (Brasil, 2015).

\subsection{Condições meteorológicas para o episódio de seca mais extremo (março/2013)}

As Figs. 10 e 11 apresentam, respectivamente, a anomalia espacial de precipitação (em $\mathrm{mm}$ ) e a distribuição espacial do SPI sobre a Sub-Bacia Choró nas escalas SPI-3 (Fig. 11A), SPI-6 (Fig. 11B) e SPI-12 (Fig. 11C), para março de 2013-mês que registrou o episódio de seca mais extremo, dentre todas as escalas analisadas, conforme mostrado na Fig. 3C.

Neste mesmo ano, o Ceará enfrentou a sua sétima pior seca desde 1950 (Carmo e Lima, 2020). Conforme mostra a Fig. 10, a região analisada enfrentou grandes déficits pluviométricos registrando chuvas abaixo da média histórica. Fato que justifica os SPI-3 e SPI-6 categorizarem de Normal a Extremamente Seco os eventos de seca identificados em março de 2013 dentro da Sub-Bacia Choró, como mostrado na Fig. 11.

A Fig. 12 mostra a anomalia da TSM referente as regiões NINO3.4 (A) e TNAI e TSAI (B) em março de 2013.

Em relação ao fenômeno ENOS, foram identificadas condições de neutralidade no Oceano Pacífico Equatorial, o que indica a maior contribuição da configuração do Oceano Atlântico Tropical sobre a escassez de chuvas no norte do NE. De acordo com a NOAA, o ONI apresentou valores suaves de -0.3 , o que configura um período Normal, enfatizada por anomalias negativas suaves de TSM, da ordem de -0.5 a $-1{ }^{\circ} \mathrm{C}$ sobre a região NINO3.4. O evento extremo de seca pode ter tido sua causa associada ao alcance de uma posição da ZCIT mais ao norte, fato, que por sua vez, esteve relacionado ao gradiente meridional de TSM nas áreas tropicais do Oceano Atlântico, onde persistiram águas superficiais anomalamente aquecidas no TNAI. É sabido, que neste período o alcance da ZCIT mais ao norte/sul de sua posição climatológica é determinante na qualidade de chuvas sobre o norte do NEB (Uvo, 1989; Molion e Bernardo, 2002).

No geral, em um breve estudo realizado por Silva et al. (2019), condições semelhantes foram observadas na análise do SPI também para a Sub-Bacia Choró, considerando escalas superiores às adotadas no presente tra- 

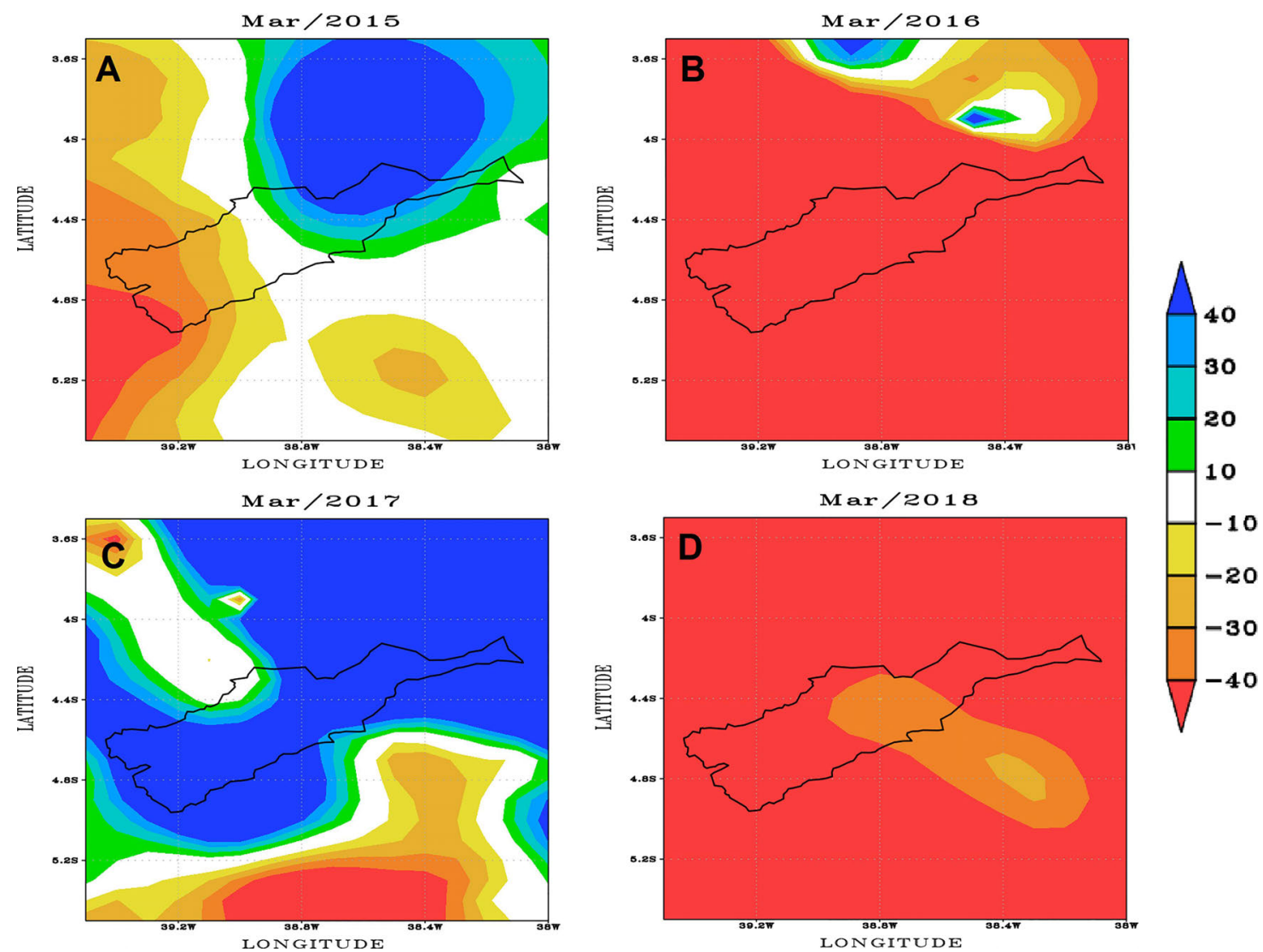

Figura 8 - Anomalia de Precipitação (em mm) para o mês de março nos anos 2015 (A) 2016 (B) 2017 (C) 2018 (D). De acordo com a média climatologica mensal de Janeiro de 1974 a Dezembro de 2018.
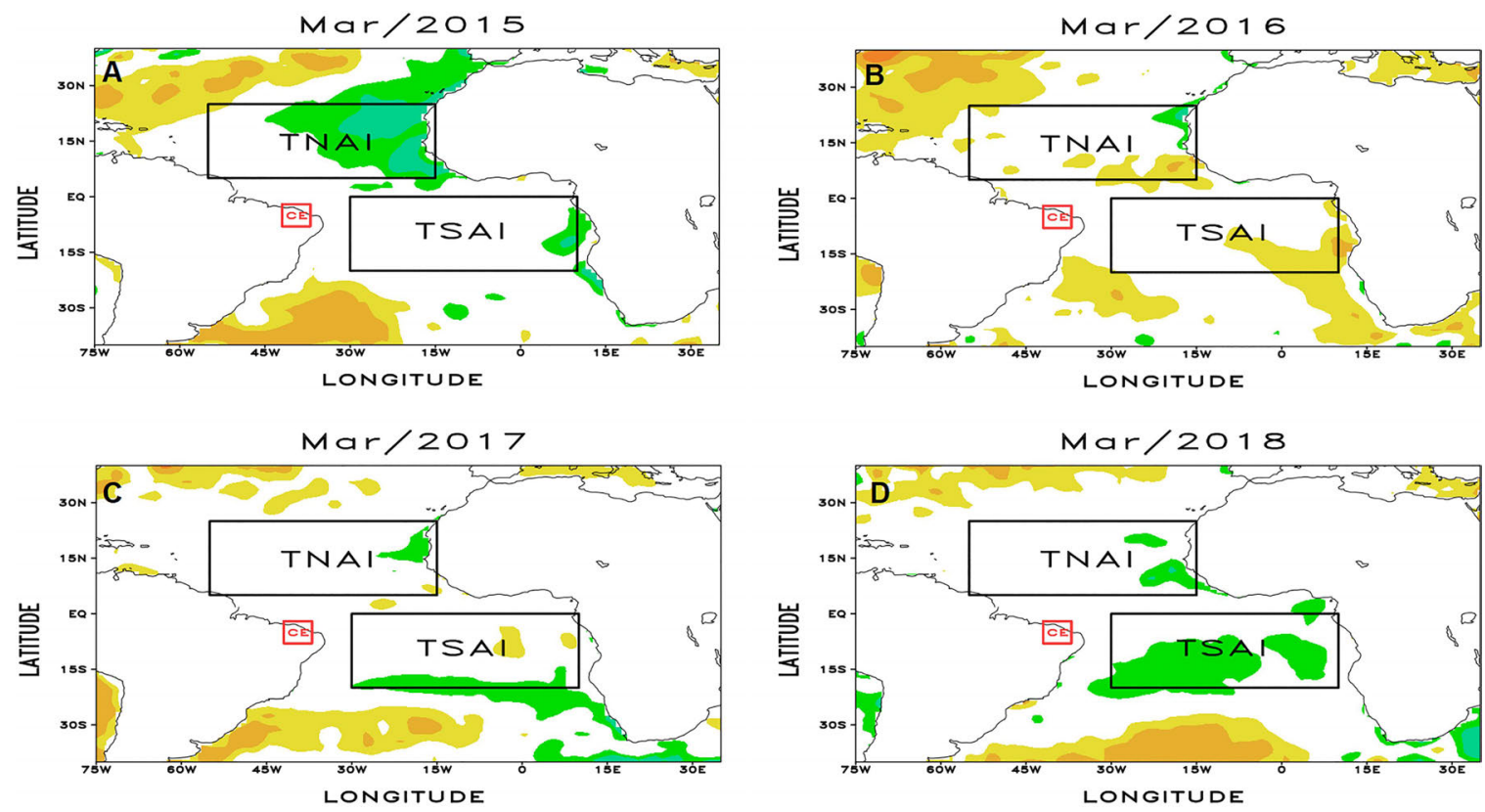

Figura 9 - Anomalia da TSM referente ao TNAI e TSAI, para o mês de março nos anos 2015 (A) 2016 (B) 2017 (C) 2018 (D). De acordo com a média climatológica mensal de Janeiro de 1971 a 2000 . Unidade em ${ }^{\circ} \mathrm{C}$. 


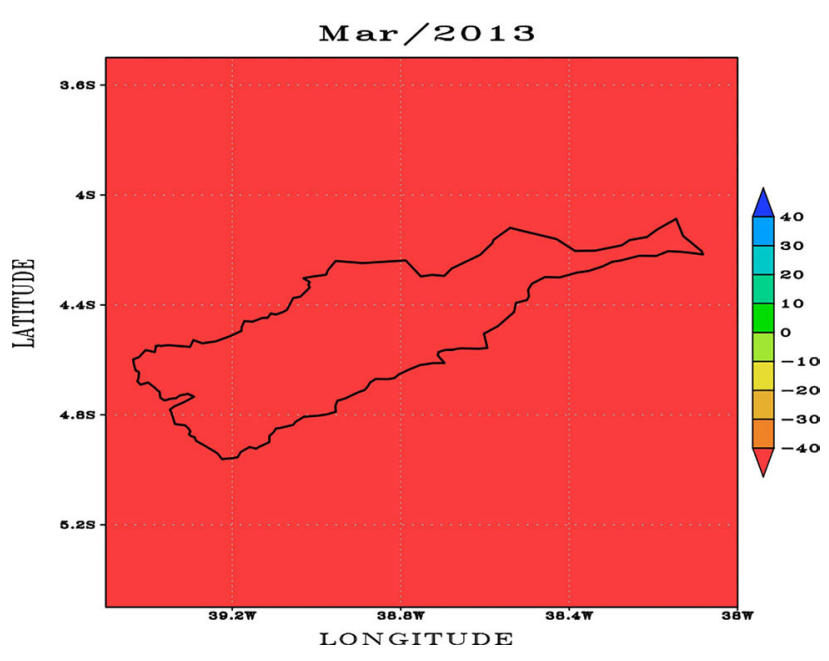

Figura 10 - Anomalia de Precipitação (em mm) para março de 2013. De acordo com a média climatologica mensal de Janeiro de 1974 a Dezembro de 2018.

balho. Os autores identificaram eventos secos de maior intensidade em 2013 e 2016, tendo neste último forte impacto na disponibilidade hídrica da região, o que acarretou em grandes prejuízos nos setores agrícolas, socioambientais e pecuários nos municípios que compõem a bacia supracitada.

\section{Conclusões}

Através do cálculo do SPI na Sub-Bacia Choró, observou-se uma acentuada variabilidade interanual desse índice. Uma maior ocorrência de eventos de seca foram identificados entre os anos de 2012 a 2018, com melhor representação nas escalas interanuais de SPI, considerando-se a intensidade e duração. Além disso, se constatou uma relação entre os períodos contendo eventos secos (úmidos), de maior intensidade, e os períodos que registraram a ocorrência de fenômenos de El Niño Oscilação Sul (La Niña), o que reforça, na maioria dos casos analisados, a influência dos eventos de circulação de grande escala sobre a região de estudo. Ademais, a configuração do Oceano Atlântico Tropical e o posicionamento da ZCIT contribuíram para os déficits de chuva observados sobre a bacia e, consequentemente, a maioria dos episódios de seca identificados neste estudo.

O SPI se mostrou uma ferramenta de grande utilidade no que tange a quantificação de eventos secos e chuvosos na Sub-Bacia Choró, podendo ser útil no monitoramento do déficit hídrico em escalas temporais de curto, médio e longo prazo. Recomenda-se para trabalhos futuros, uma análise mais robusta, oferecendo subsídios matemáticos, de forma a correlacionar a variação dos fenômenos ENOS (bem como outros fenômenos de
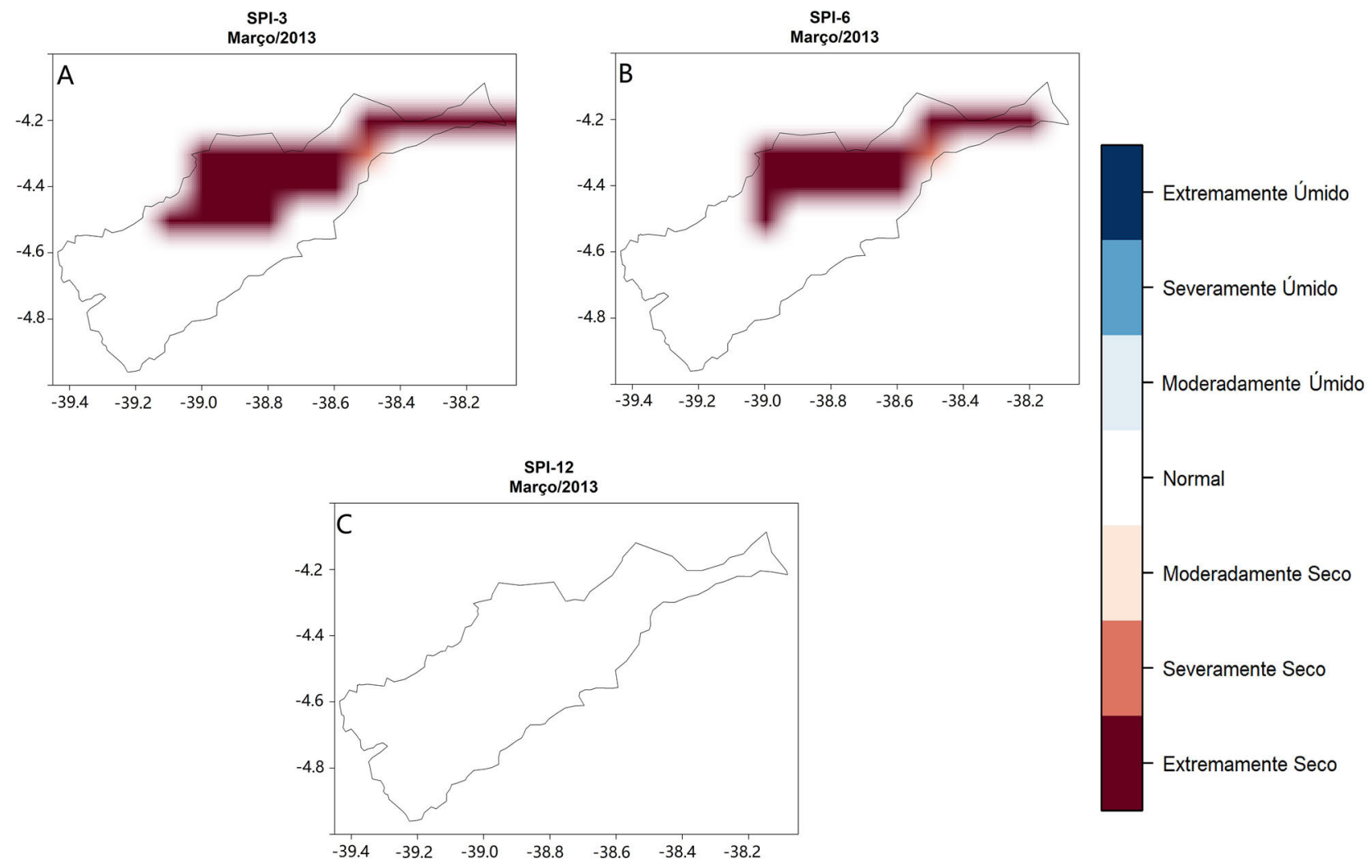

Normal

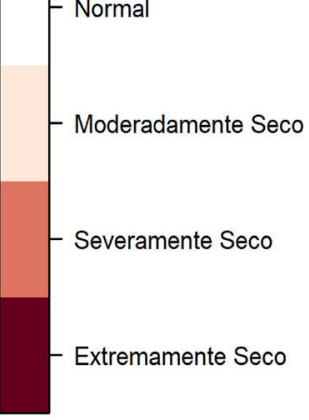

Figura 11 - Variabilidade Espacial do SPI sobre a Bacia do Rio Choró em março de 2013 nas escalas SPI-3 (A) SPI-6 (B) SPI-12 (C). 

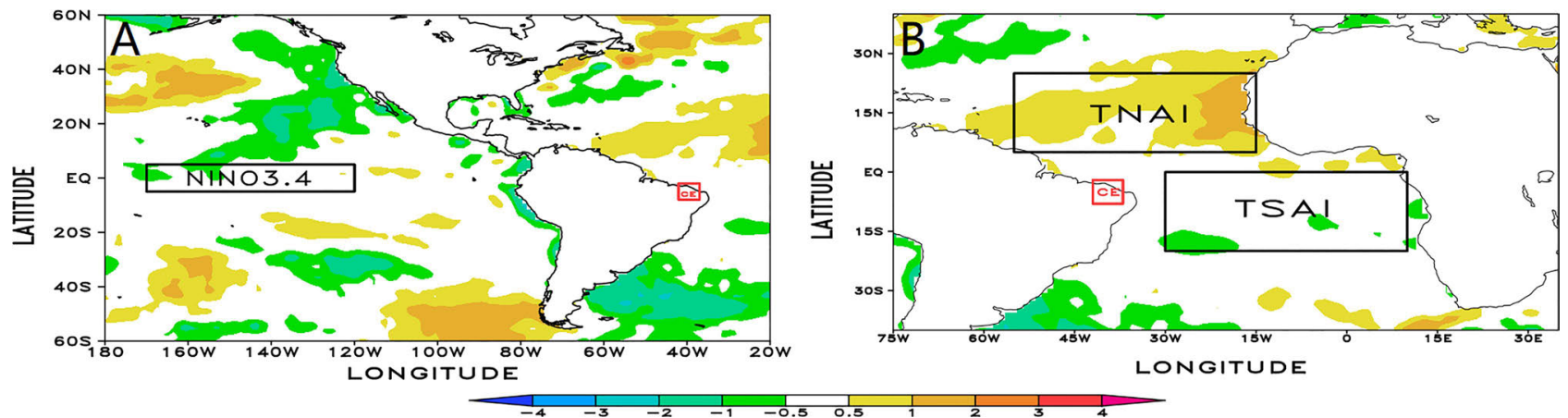

Figura 12 - Anomalia da TSM referente as regiões NINO3.4 (A) e TNAI e TSAI (B), no mês de março para o ano 2013. De acordo com a média climatológica mensal de Janeiro de 1971 a 2000 . Unidade em ${ }^{\circ} \mathrm{C}$.

pequena e grande escala) aos eventos extremos de secas e chuvas. Constatada essa correlação, motiva-se a construção de uma rede de monitoramento podendo fornecer condições básicas para a tomada de decisões diante das frequentes variações sazonais e interanuais do clima, subsidiando o planejamento, previsão e/ou mitigação dos efeitos de eventos intensos de seca sobre sobre a referida região e ecossistema bem como o conseguinte avanço de secas meteorológicas para secas agrícola e hidrológica.

\section{Agradecimentos}

O presente trabalho foi realizado com apoio da Coordenação de Aperfeiçoamento de Pessoal de Nível Superior, Brasil (CAPES) - Código de Financiamento 001 - e Fundação Cearense de Apoio ao Desenvolvimento Científico e Tecnológico (FUNCAP).

\section{Referências}

AGÊNCIA NACIONAL DAS ÁGUAS (ANA). Monitor de Secas. Disponível em http://monitordesecas.ana.gov.br/, acesso em 8 abr. 2020.

AGHAKOUCHAK, A.; FARAHMAND, A.; MELTON, F.S.; TEIXEIRA, J.; ANDERSON, M.C.; WARDLOW, B.D.; HAIN, C.R. Remote Sensing of Drought: Progress, Challenges and Opportunities. Rev. Geophys., v. 53, n. 2, p. 452-480, 2015.

ALBUQUERQUE, T.M.A. Estudo dos Processos de Gestão de Seca: Aplicação no Estado do Rio Grande do Sul. Tese de Doutorado em Recursos Hídricos e Saneamento Ambiental, Universidade Federal do Rio Grande do Sul, Porto Alegre, 406 p., 2010.

ALLEN, S.; BARROS, V.; BURTON, I.; CAMPBELL-LENDRUM, D.; CARDONA, O.; CUTTER, S.; WILBANKS, T. Summary for Policymakers. Managing the Risks of Extreme Events and Disasters to Advance Climate Change Adaptation: Special Report of the Intergovernmental Panel on Climate Change. Cambridge: Cambridge University Press, p. 3-22, 2012.

ALIZADEH-CHOOBARI, O. Contrasting Global Teleconnection Features of the Eastern Pacific and Central Pacific El
Niño Events. Dynamics of Atmospheres and Oceans, v. 80, n. 4, p. 139-154, 2017.

BONFIM, O.E.T.; DA SILVA, D.F. Aplicação do Índice de Precipitação Normalizada nos Casos de ENOS Canônico e Modoki ocorridos na Bacia Hidrográfica Aguapeí-Peixe/ SP. Revista Ibero-Americana de Ciências Ambientais, v. 9, n. 3, p. 83-94, 2018.

BRASIL. Boletim de Informações Climáticas: Fenômeno EI Niño se Configura no Oceano Pacífico Equatorial. Infoclima. Disponível em http://infoclima.cptec.inpe.br/ rinfo/ pdf_infoclima/201503.pdf, acesso em 21 jan. 2021.

BRASIL. Boletim de Informações Climáticas: O Verão Termina com Grande Irregularidade na Distribuição das Chuvas sobre o Brasil. Disponível em http://infoclima. cptec.inpe.br/ rinfo/pdf_infoclima/201603.pdf, acesso em 21 jan. 2021.

BRASIL. Boletim de Informações Climáticas: A Estação Chuvosa do Norte da Região Nordeste Terminará com Déficit Pluviométrico e Hídrico. Disponível em http://info clima.cptec.inpe.br/ rinfo/pdf_infoclima/201703.pdf, acesso em 21 jan. 2021.

BRASIL. Boletim de Informações Climáticas: Fenômeno La Niña Inicia Processo de Declínio no Pacífico Equatorial. Disponível em http://infoclima.cptec.inpe.br/ rinfo/pdf_in foclima/201803.pdf, acesso em 21 jan. 2021.

BRASIL. Condições da Última Semana: Monitoramento do El Niño Durante NDJ-2019/2020, Fev. 2020. Disponível em http://enos.cptec.inpe.br/, acesso em 21 jan. 2021.

BRITO, S.S.B.; CUNHA, A.P.M.A.; CUNNINGHAM, C.C.; ALVALÁ, R.C.; MARENGO, J.A.; CARVALHO, M.A. Frequency, Duration and Severity of Drought in the Semiarid Northeast Brazil Region. International Journal of Climatology, v. 38, n. 2, p. 517-529, 2017.

CARMO, M.V.N.S.; LIMA, C.H.R. Caracterização Espaço-Temporal das Secas no Nordeste a partir da Análise do Índice SPI. Rev. Bras. Meteorol., v. 35, n. 2, p. 233-242, 2020.

CEARÁ. Relatório de Diagnóstico Ambiental das Bacias Metropolitanas: Elaboração do Plano de Segurança Hídrica das Bacias Hidrográficas Estratégicas do Acaraú, Metropolitanas e da Sub-Bacia do Salgado. Fortaleza: Secretaria de Recursos Hídricos, 128 p., 2016.

DA SILVA, D., LIMA, M., SOUZA NETO, P., GOMES, H., SILVA, F., ALMEIDA, H., COSTA, R.; PEREIRA, M. Caracterização de Eventos Extremos e de suas Causas Cli- 
máticas com Base no Índice Padronizado de Precipitação Para o Leste do Nordeste. Revista Brasileira de Geografia Física, v. 13, n. 2, p. 449-464, 2020.

DOS SANTOS, S.R.Q.; CUNHA, A.P.M.A.; RIBEIRO-NETO, G.G. Avaliação de Dados de Precipitação para o Monitoramento do Padrão Espaço-Temporal da Seca no Nordeste do Brasil. Revista Brasileira de Climatologia, v. 25, n. 2, p. 80-100, 2019.

FERNANDES, D.S.; HEINEMANN, A.B.; PAZ, R.L.; AMORIN, A.O.; CARDOSO, A.S. Índices para a Quantificação da Seca. Santo Antônio de Goiás: Embrapa Arroz e Feijão, 48 p., 2009.

GOIS, G.; DELGADO, R.C.; OLIVEIRA-JÚNIOR, J.F. Modelos Teóricos Transitivos Aplicados na Interpolação Espacial do Standardized Precipitation Index (SPI) para os Episódios de El Niño Forte no Estado do Tocantins, Brasil. Irriga, v. 20, n. 2, p. 371-387, 2015.

GUTTMAN, N.B. Accepting the standardized precipitation index: A calculation algorithm. Journal of the American Water Resources Association, v. 35, n. 2, p. 311-322, 1999.

INSTITUTO BRASILEIRO DE GEOGRAFIA E ESTATÍSTICA. Mapa de Clima do Brasil. Disponível em https:// www.ibge.gov.br/geociencias/informacoes-ambientais/ 15817-clima.html?=\&t=acesso-ao-produto, acesso em 15 jan. 2021.

KÖPPEN, G.; GEIGER, M. Das Geographische System der Klimate. Handbuch der Klimatologie: Berlin, 44 p., 1936.

MARENGO, J.A.; CUNHA, A.P.M.A.; NOBRE, C.A.; RIBEIRO NETO, G.G.; MAGALHAES, A.R.; TORRES, R.R.; SAMPAIO, G.; ALEXANDRE, F.; ALVES, L.M.; CUARTAS, L.A.; DEUSDARÁ, K.R.L.; ÁLVALA, R.C.S. Assessing drought in the drylands of Northeast Brazil under regional warming exceeding $4{ }^{\circ} \mathrm{C}$. Nat. Hazards, v. 103 , n. 1 , p. $2589-2611,2020$.

MCKEE, T.B.; DOESKEN, N.J.; KLEIST, J. The relationship of drought frequency and duration to time scales. Proceedings Eighth Conference on Applied Climatology. Anahaim: AMS, p. 179-184, 1993.

MCKEE, T.B.; DOESKEN, N.J.; KLEIST, J. Drought monitoring with multiple time scales. Proceedings Ninth Conference on Applied Climatology. Boston: AMS, p. 233-236, 1995.

MOLION, L.C.B.; BERNARDO, S.O. Uma Revisão da Dinâmica das Chuvas no Nordeste Brasileiro. Revista Brasileira Meteorologia, v. 17, n. 1, p. 1-10, 2002.

NASCIMENTO, F.; BRAGA, C.; ARAÚJO, F.; COSTA, E. Caracterização de eventos secos e chuvosos na microrregião de Imperatriz - MA. Revista Brasileira de Geografia Física, v. 8, n. 2, p. 325-333, 2015.

NASCIMENTO, F.C.A.; BRAGA, C.C.; ARAUJO, F.R.C.D. Análise Estatística dos Eventos Secos e Chuvosos de Precipitação do Estado do Maranhão. Rev. Bas. Meteorol., v. 32, n. 3, p. 375-386, 2017.

NOBRE, P.; SHUKLA, J. Variations of sea surface temperature, wind stress, and rainfall over the Tropical Atlantic and South America. J. Climate, v. 9, n. 10, p. 2464-2479, 1996.

NATIONAL OCEANIC AND ATMOSPHERIC ADMINISTRATION. Equatorial Pacific Sea Surface Temperatures., Nov. 2017. Disponível em https://www.ncdc.noaa. gov/teleconnections/enso/indicators/sst.php, acesso em 10 jan. 2021.

PONTES FILHO, J.D.; DE SOUZA FILHO, F.A.; MARTINS, E.S.P.R.; STUDART, T.M.C. Copula-based multivariate frequency analysis of the 2012-2018 drought in Northeast Brazil. Water., v. 12, n. 3, p. 834, 2020.

R CORE TEAM. R: A Language and Environment for Statistical Computing. R Foundation for Statistical Computing, Vienna, Austria, 2020.

REBOITA, M.S.; SANTOS, I. A. Influência de alguns padrões de teleconexão na precipitação no Norte e Nordeste do Brasil. Revista Brasileira de Climatologia, v. 15, n. 10, p. 29-47, 2014.

SANTOS, S.R..; BRAGA, C. C.; SANSIGOLO, C.A.; SANTOS, A.P.P. Determinação de regiões homogêneas do Índice de Precipitação Normalizada (SPI) na Amazônia Oriental. Rev. Bras. Meteorol., v. 32, n. 1, p. 111-122, 2017.

SILVA, G.K.; MARCOS JUNIOR, A.D.; SILVA, M.V.M.; SILVEIRA, C.S. Dinâmica dos múltiplos usos da água em períodos de escassez hídrica: uma análise para Bacia do rio Choró, Ceará, Brasil. Anais $2^{\circ}$ Simpósio Brasileiro de Recursos Hídricos. Porto Alegre: ABRH, 2019.

SINGH, P.; GNANASEELAN, C.; CHOWDARY, J.S. NorthEast monsoon rainfall extremes over the southern peninsular India and their association with El Niño. Dynamics of Atmospheres and Oceans, v. 80, n. 4, p.1-11, 2017.

SOUSA, F.A.S.; MACEDO, M.J.H.; GUEDES, R.V.S.; SILVA, V.P.R. O Índice de Precipitação Padronizada (IPP) na identificação de extremos de chuvas e secas na Bacia do Rio Paraguaçu (BA). Revista Ambiência, v. 12, n. 2, p. 707 719, 2016.

TEDESCHI, R.G.; GRIMM, A.M.; CAVALCANTI, I.F. A. Influence of Central and East ENSO on precipitation and its extreme events in South America during Austral autumn and winter. International Journal of Climatology, v. 36, n. 15, p. 4797-4814, 2016.

TRENBERTH, K.E.; HOAR, T.J. El Niño and climate change. Geophysical Research Letters, v. 24, n. 23, p. 3057-3060, 1997.

ULIANA, E.M.; DOS REIS, E.F.; DE SOUZA, A.P.; DA SILVA, J.G.F.E; XAVIER, A.C. Utilização do índice de precipitação padronizado para a região Norte do Estado do Espírito Santo. Revista Irriga, v. 20, n. 3, p. 414-428, 2015.

UVO, C. A Zona de Convergência e Sua Relação com a Precipitação no Nordeste Brasileiro. Dissertação de Mestrado em Meteorologia, Instituto Nacional de Pesquisas Espaciais, São José dos Campos, 120 p., 1989.

VICENTE-SERRANO, S.M.; BEGUERIA, S.; LÓPEZ-MORENO, J.I. A multiscalar drought index sensitive to global warming: The standardized precipitation evapotranspiration index. J. Climate, v. 23, n.7, p. 1696-1718, 2010.

ZARGAR, A.; SADIQ, R.; NASER, B.; KHAN, F.I. A review of drought indices. Environ. Rev., v. 19, n. 1, p. 333-349, 2011.

License information: This is an open-access article distributed under the terms of the Creative Commons Attribution License (type CC-BY), which permits unrestricted use, distribution and reproduction in any medium, provided the original article is properly cited. 